\title{
Aus dem VSAS wird ScolarMed CH
}

\author{
Annemarie Tschumpera, Ursula Kälin-Kellera, Cristina Fiorini Bernasconi ${ }^{b}$, für ScolarMed CH \\ ${ }^{\text {a }}$ Co-Präsidentinnen von ScolarMed CH; ${ }^{b}$ Vorstandsmitglied von ScolarMed CH
}

An der Mitgliederversammlung vom 7. Juni 2018 wurde die Vereinigung der Schulärztinnen und Schulärzte der Schweiz (VSAS) in die Fachorganisation ScolarMed CH für alle im Schulgesundheitsdienst tätigen medizinischen Fachpersonen überführt. Die Organisation will die Medizin auch in Zukunft als tragenden Fachbereich der Schulgesundheitsdienste verankern und zur Sicherung einer bedarfsgerechten schulärztlichen Versorgung aller Schülerinnen und Schüler beitragen.

Seit über 100 Jahren unterstützt der schulärztliche Dienst als bewährter öffentlicher medizinischer Dienst die Schulen dabei, allen Kindern die Entfaltung ihres Bildungspotentials zu ermöglichen und ihre Gesundheit über die ganze Schulzeit zu fördern. Ihm fallen, kantonal vorgegeben, folgende wichtige Aufgaben zu:

- Beratung der Schule bei gesundheitlichen Fragestellungen im Kontext der schulischen Entwicklung einzelner Schülerinnen und Schüler, so beispielsweise bei chronischer Krankheit, Absentismus, Entwicklungsauffälligkeiten, Kindeswohlgefährdung etc.;

- Beratung der Schule bezüglich bedarfsgerechter Ausgestaltung der schulischen Gesundheitsförderung sowie betriebsärztlicher Fragen (Hygiene, Notfallorganisation etc.);

- Bekämpfung von Infektionskrankheiten, Kontrolle der Impfdokumente gemäss Epidemiengesetz sowie Impfungen und Impfaktionen in Schulen (z.B. gegen HPV);

- Durchführung schulärztlicher Untersuchungen mit der spezifischen Möglichkeit, sonst nicht oder ungenügend versorgte Kinder zu erfassen und nachsuchend zu betreuen.

Der schulärztliche Dienst ergänzt die kinder- und hausärztliche Betreuung, indem er sowohl die Gesundheit des einzelnen Schulkindes als auch die Gesundheit des Schülerkollektivs im Auge hat. Kollektiv auftretende Probleme können so mit der Schule auch kollektiv angegangen werden. Bereits seit Jahren erfassen beispielsweise einige hauptamtliche Dienste gemeinsam anonymisiert Daten zum BMI, woraus Massnahmen wie Purzelbaum-Kindergärten und Ernährungsprogramme begründet werden konnten.

Dank seiner Nähe zur Schule und seiner engen Zusammenarbeit mit den behandelnden Ärztinnen und Ärz- ten erfüllt der schulärztliche Dienst oft eine Brückenfunktion zwischen medizinischer Behandlung, Schule, Kind und Eltern. So können medizinisch notwendige Unterstützungsmassnahmen in der Schule mit Rücksicht auf die schulische Entwicklung des Kindes optimal umgesetzt werden.

Im schulärztlichen Dienst arbeiten drei Berufsgruppen eng zusammen:

- Haupt- und nebenamtliche Schulärztinnen und Schulärzte verantworten den schulärztlichen Dienst und die schulärztliche Versorgung der Schülerinnen und Schüler. Sie beraten Schulleitungen, Lehrpersonen und Eltern, klären Kinder mit schulbezogenen Fragestellungen ab, führen die kantonal vorgegebenen Untersuchungen sowie Impfungen durch und überwachen die gesundheitliche Situation in den Schulen (Gesundheit des Schülerkollektivs, Hygiene, Organisation von Notfallmassnahmen etc.).

- Schulpflegefachpersonen sind vor allem in der Westschweiz, aber zunehmend auch in der Deutschschweiz erste Ansprechpersonen für schulbezogene medizinische Fragen. Sie sind in den Schulen präsent, übernehmen in Zusammenarbeit mit den Schulärztinnen und -ärzten einen Teil der schulärztlichen Screenings und Beratungen und arbeiten in Gesundheitsförderungsprojekten der Schulen mit.

- Medizinische Praxisfachpersonen kennen über ihre organisatorischen Aufgaben die Schulen besonders gut und können wesentlich zu einer guten Vertrauensbasis zwischen Schule, Familie und schulärztlichem Dienst beitragen. Bei entsprechender Schulung und Fortbildung können sie zudem vermehrt auch delegierte Aufgaben übernehmen wie Screenings oder Beratungen zu gewissen Themen. 
Die haupt- und nebenamtlich im schulärztlichen Dienst tätigen Fachpersonen bilden sicher die grösste «Public Health Workforce» der Schweiz. Bereits 1899 wurde die Schweizerische Gesellschaft für Schulgesundheitspflege gegründet. Die Gesellschaft wurde im Verlauf der Jahre und über diverse Zwischenschritte und Fusionen mit Partnerorganisationen 2005 schliesslich zu Public Health Schweiz. ScolarMed $\mathrm{CH}$ ist heute dort assoziierte Fachgesellschaft. Mit dem Schritt von der ärztlichen Fachorganisation hin zur interprofessionellen Vereinigung aller medizinischen Fachpersonen der Schulgesundheitsdienste geht der schulärztliche Dienst einmal mehr voran und trägt bereits heute der aktuellen Entwicklung von der vorwiegend ärztlichen hin zur interprofessionell orientierten medizinischen Versorgung auch institutionell Rechnung.

Die Fachvereinigung ScolarMed $\mathrm{CH}$ verfolgt drei zentrale Zielrichtungen:

- ScolarMed CH macht sich stark für die Gesundheit von Schülerinnen und Schülern sowie für die Sicherstellung des Zugangs zu kompetenter schulärztlicher Versorgung für alle Schülerinnen und Schüler in der Schweiz.

- ScolarMed CH setzt sich ein für eine hohe fachliche Qualität der schulärztlichen Arbeit aller beteiligten medizinischen Berufsgruppen. Der Einsatz im schulärztlichen Dienst erfordert Fachwissen in verschiedensten Bereichen: von Pädiatrie und Entwicklung, Infektiologie und Vakzinologie über Pädagogik, Lehrpläne und Schulorganisation bis zu Grundlagen von Public Health, Arbeitsmedizin sowie Ansät- zen der Gesundheitsförderung und Prävention. Mit Angeboten der Weiter- und Fortbildung, teilweise in Zusammenarbeit mit Universitäten und Fachhochschulen, einer Website www.scolarmed.ch (ergänzt mit vertiefenden fachlichen Beiträgen für Mitglieder auf dem geschützten Teil der Website) bis hin zur Erarbeitung fachlicher Standards will die Vereinigung dazu beitragen, dass die schulärztliche Arbeit «state of the art» geleistet wird.

- Schliesslich bezweckt ScolarMed CH als Vereinigung der in den schulärztlichen Diensten haupt- oder nebenamtlich tätigen Fachpersonen die Förderung der Zusammenarbeit, des fachlichen Austausches und der beruflichen Identität ihrer Mitglieder.

Wir freuen uns, möglichst viele schulärztlich tätige Ärztinnen und Ärzte, medizinische Praxisfachpersonen und Schulpflegefachpersonen aus allen Landesregionen für eine Mitgliedschaft zu gewinnen und so ScolarMed CH zu stärken. Als Mitglied haben Sie über den geschützten Teil der Webseite von ScolarMed $\mathrm{CH}$ Zugang zu vielfältigen, praxisrelevanten Fachinformationen, die Möglichkeit, sich über die Mitgliederdatenbank mit Kolleginnen und Kollegen zu vernetzen und sich mit Fragen und Anliegen an den Vorstand von ScolarMed CH zu wenden. Sie werden zudem regelmässig über schulärztliche Weiterbildungen und sonstige Aktualitäten informiert.

Wir laden Sie ebenfalls herzlich ein, an unserer nächsten nationalen Tagung teilzunehmen. Sie wird am 28.11.2019 in St. Gallen stattfinden. Zusätzliche Informationen finden Sie auf unserer Website www.scolarmed.ch 